\title{
Quintessential inflation with a trap and axionic dark matter
}

\author{
Konstantinos Dimopoulos $\odot,{ }^{1, *}$ Mindaugas Karčiauskas, ${ }^{2, \dagger}$ and Charlotte Owen ${ }^{1,+}$ \\ ${ }^{1}$ Consortium for Fundamental Physics, Physics Department, Lancaster University, \\ Lancaster LA1 4YB, United Kingdom \\ ${ }^{2}$ Departamento de Física Teórica and Instituto de Física de Partículas y del Cosmos IPARCOS, \\ Universidad Complutense de Madrid, E-28040 Madrid, Spain
}

(Received 18 July 2019; published 18 October 2019)

\begin{abstract}
We study a new model of quintessential inflation which is inspired by supergravity and string theory. The model features a kinetic pole, which gives rise to the inflationary plateau, and a runaway quintessential tail. We envisage a coupling between the inflaton and the Peccei-Quinn (PQ) field which terminates the roll of the runaway inflaton and traps the latter at an enhanced symmetry point (ESP), thereby breaking the PQ symmetry. The kinetic density of the inflaton is transferred to the newly created thermal bath of the hot big bang due to the decay of PQ particles. The model successfully accounts for the observations of inflation and dark energy with natural values of the model parameters, while also resolving the strong $C P$ problem of $\mathrm{QCD}$ and generating axionic dark matter, without isocurvature perturbations. Trapping the inflaton at the ESP ensures that the model does not suffer from the infamous 5 th force problem, which typically plagues quintessence.
\end{abstract}

DOI: 10.1103/PhysRevD.100.083530

\section{INTRODUCTION}

By now we have most of the history of the Universe figured out. The hot big bang model covers pretty much the entire timeline, from a few seconds after the original explosion until the present time, almost. However, the edges of the story are still unclear, because observations suggest that the Universe is undergoing accelerated expansion at very early and very late times and such expansion cannot be part of the hot big bang, where the Universe is filled with relativistic and nonrelativistic matter only. General relativity dictates that accelerated expansion can occur only if the Universe is filled by an exotic substance, with negative enough pressure. For the early Universe, the required substance is usually taken as one (or more) potentially dominated scalar field and the accelerated expansion phase is cosmic inflation [1-4] and the scalar is called the inflaton field. Inflation sets the initial conditions of the hot big bang. For the late Universe however, the simplest explanation is vacuum density, due to a nonzero value of the cosmological constant $\Lambda$.

\footnotetext{
*konst.dimopoulos@lancaster.ac.uk †mindaugas.k@ucm.es

"c.owen@lancaster.ac.uk
}

Published by the American Physical Society under the terms of the Creative Commons Attribution 4.0 International license. Further distribution of this work must maintain attribution to the author(s) and the published article's title, journal citation, and DOI. Funded by SCOAP.
The cosmological constant has to be there but what is its value? The most natural choice is given by the Planck scale, because this is the only scale in general relativity and also because this is the cutoff scale of particle theory (beyond this the theory breaks down). This however is at odds with nature. This cosmological constant problem [5] predates the observations of recent accelerated expansion. The way it used to be addressed is by assuming that the cosmological constant is set to zero by some unknown symmetry. Once late accelerated expansion was observed, many authors suggested that this amounted to observing the true value of the cosmological constant. The problem is that the associated vacuum density has to be comparable to the density of the Universe today, which is about 120 orders of magnitude smaller than the "natural" value of $\Lambda$. This has been called (by Lawrence Krauss) "the worst finetuning in physics". To avoid this fine tuning, it was suggested that, as in inflation, the current accelerated expansion is due to another potentially dominated scalar field, called quintessence; the fifth element after baryons, dark matter, photons and neutrinos [6-8]. It is important to point out that the quintessence proposal does not solve the cosmological constant problem because $\Lambda$ is still assumed to be zero due to some unknown symmetry.

Since they are both based on the same idea, it is natural to unify the two in quintessential inflation $[9,10]$ (for a recent list of references see $[11,12]$ and $[13,14])$, which considers that the inflaton field survives until today and becomes quintessence. Apart from being economic, quintessential inflation attempts to treat the early and late accelerated 
expansion phases in a single theoretical framework. Moreover, there are some practical advantages as well; for example the initial conditions of quintessence are determined by the inflationary attractor.

A scalar potential which satisfies all the requirements of inflation and dark energy observations is hard to formulate because of the $\sim 110$ orders of magnitude difference in energy density between the inflationary energy scale and the energy scale of dark energy today. To incorporate such a large difference in energy density scales often requires a very curved scalar potential [15], which produces inflationary predictions incompatible with the Planck satellite observations [16]. Moreover, after inflation, the field rolls down the incredibly steep potential and gains so much kinetic energy that it is transposed many Planckian distance in field space. This means the potential is at risk from UV corrections, throwing the predictability of the theory into question and destabilising the flatness of the potential.

In this paper, to overcome the above problems, we start with an exponential potential with a noncanonical kinetic term, featuring a pole at the origin, which can be theoretically motivated e.g., in supergravity theories. We utilize a field redefinition to regain canonical kinetic terms and thereby transpose the pole to infinity. Doing so, introduces a plateau into the potential, ensuring inflationary observables match the observations of the Planck satellite (which favors a plateau inflation model). The model naturally features a quintessential tail. To ensure the validity of our setup, we stop the roll of the inflaton field by trapping it at an enhanced symmetry point (ESP) before it travels over a super-Planckian distance in field space. We demonstrate in detail that, through this trapping, we can transform the kinetic density of the field into the radiation density of the hot big bang, reheating the Universe.

Reheating in quintessential inflation is a challenging issue, because it cannot occur through inflaton decay (as is otherwise typical in inflation) because the inflaton must survive until the present and become quintessence. A number of reheating mechanisms have been put forward, the most important of which are gravitational reheating $[17,18]$, instant preheating $[19,20]$, curvaton reheating $[21,22]$ and recently nonminimal reheating [23] (also called Ricci reheating [24]). Reheating the Universe through trapping the runaway inflaton is a novel mechanism, although the trapping mechanism has been considered before in quintessential inflation [25,26], but only for being responsible for the inflation part of the scenario as in trapped inflation [27].

In the spirit of economy, aligned with the philosophy behind quintessential inflation, we consider that the ESP is due to a coupling of the inflaton direction with the PecceiQuinn field [28,29], so that after trapping, the Peccei-Quinn phase transition confines the inflaton and generates a large inflaton mass such that there is no threat of violation of the equivalence principle (5th force problem), which typically is a problem with quintessence [30-32]. The field remains trapped with nonzero potential density, which explains the dark energy observations, while the theory also incorporates the QCD axion, which can be the dark matter [33].

We use natural units where $c=\hbar=k_{\mathrm{B}}=1$ and $8 \pi G=m_{P}^{-2}$, where $m_{P}=2.43 \times 10^{18} \mathrm{GeV}$ is the reduced Planck mass.

\section{THE MODEL}

We start with a Lagrangian density well motivated in both supergravity and string theory ${ }^{1}$ with a perturbative and nonperturbative part:

$$
\begin{aligned}
\mathcal{L}= & \frac{\alpha}{2}\left(\frac{m_{\mathrm{Pl}}}{\phi}\right)^{2}(\partial \phi)^{2}+\frac{(\partial \chi)^{2}}{2}-V_{0} e^{-\kappa \phi / m_{\mathrm{Pl}}} \\
& -V(\chi)-\frac{g^{2}}{2}\left(\phi-\phi_{\mathrm{ESP}}\right)^{2} \chi^{2}
\end{aligned}
$$

The $\chi$ field is taken to be the Peccei-Quinn field $[28,29]$ associated with the U(1) Peccei-Quinn symmetry, whose Pseudo-Nambu-Godlstone boson is the QCD axion, which is a prominent dark matter candidate [33]. The order parameter $f$ is called the axion decay constant. In this case, we have

$$
\begin{gathered}
V(\chi)=\frac{\lambda}{4}\left(\chi^{2}-f^{2}\right)^{2}, \\
\lambda \sim 1, \\
f \sim 10^{12} \mathrm{GeV} .
\end{gathered}
$$

In fact, the most likely range for the axion decay constant is $10^{10} \mathrm{GeV} \lesssim f \lesssim 10^{12} \mathrm{GeV}$. The lower bound in this range comes from the SN1987A energy loss rate, while the upper bound is required to avoid overproduction of axions. However, this latter limit is dependent on assumptions regarding the initial axion misalignment angle [33]. In this paper we consider the estimate shown in Eq. (4), noting that this choice does not make much difference in our results.

${ }^{1}$ For example, the Kähler potential for a string modulus $T$, is $K / m_{\mathrm{Pl}}^{2}=-3 \ln (T+\bar{T})=-3 \ln \left(\sqrt{2} \phi / m_{\mathrm{Pl}}\right)$, where $T=$ $\frac{1}{\sqrt{2}}(\phi+i \sigma) / m_{\mathrm{Pl}}$, with $\phi, \sigma \in \mathbb{R}$. Then the kinetic term is given by

$$
\mathcal{L}_{\text {kin }}=K_{T \bar{T}} \partial_{\mu} T \partial^{\mu} \bar{T}=\frac{3}{2}\left(\frac{m_{\mathrm{Pl}}}{\phi}\right)^{2}\left[\frac{1}{2}(\partial \phi)^{2}+\frac{1}{2}(\partial \sigma)^{2}\right]
$$

where we considered $T+\bar{T}=\sqrt{2} \phi / m_{\mathrm{Pl}}$ and the subscripts of the Kähler potential denote differentiation. In our considerations we assume that the ESP lies at a minimum in the direction of $\sigma$. We also assume that $\sigma$ is heavy during inflation so that there are no issues with excessive non-Gaussianity or isocurvature perturbations. In this example, $\alpha=3 / 2$. 
It is important to point out that no bare cosmological constant (CC) is included in the Lagrangian density in Eq. (1). This is because an unknown symmetry is presumed to set the CC to zero, as was typically assumed even before the observations of dark energy in order to overcome the infamous "cosmological constant problem." This problem is twofold: First, general relativity may introduce a classical $\mathrm{CC}$ term in the Einstein-Hilbert action. The only mass-scale in general relativity is due to Newton's gravitational constant and is the Planck mass $8 \pi G=1 / m_{\mathrm{Pl}}^{2}$. But this cannot be the mass-scale of the $\mathrm{CC}$, so a new scale must be included which is at most $10^{-30} \mathrm{~m}_{\mathrm{Pl}}$. The problem is explaining why these two scales differ so much. Second, quantum fields introduce a contribution to vacuum energy which diverges and is presumed capped at the cutoff-scale of the theory, resulting in a CC. At the moment this is at least the supersymmetry breaking scale $>10^{-15} m_{\mathrm{Pl}}$. But this is unacceptable because observations suggest that the $\mathrm{CC}$ is at most $10^{-30} m_{\mathrm{Pl}}$, for otherwise structure formation would be inhibited. The "solution" to the $\mathrm{CC}$ problem (which predates the observation of dark energy, as we mentioned) is to assume that some unknown mechanism sets the CC to exactly zero. This is why there is no CC in our model. Then, quintessence is used to explain the dark energy observations.

To assist our intuition, we can make a field redefinition to regain a canonical kinetic term with

$$
\varphi=\sqrt{\alpha} m_{\mathrm{Pl}} \ln \left(\frac{\phi}{m_{\mathrm{Pl}}}\right),
$$

the Lagrangian density then becomes

$$
\begin{aligned}
\mathcal{L}= & \frac{(\partial \varphi)^{2}}{2}+\frac{(\partial \chi)^{2}}{2}-V_{0} \exp \left(-\kappa e^{\frac{\varphi}{\sqrt{\alpha m_{\mathrm{Pl}}}}}\right)-V(\chi) \\
& -\frac{g^{2} \phi_{\mathrm{ESP}}^{2}}{2}\left(e^{\frac{\varphi-\varphi_{\mathrm{ESP}}}{\sqrt{\alpha m_{\mathrm{Pl}}}}}-1\right)^{2} \chi^{2} .
\end{aligned}
$$

The inflaton potential now features a double exponential providing a bridge between the vastly different energy scales of inflation and dark energy. The model has a pole at $\phi=0$, which is transposed to $\varphi=-\infty$ by the field redefinition, and a plateau appears in the scalar field potential, providing the perfect location for slow-roll inflation. ${ }^{2}$

After inflation has completed, the field enters a period of kination [34,35], where the dominant contribution to its energy density is its kinetic energy, the field is oblivious to the potential during this time and standard kination equations can be used [11]. After a brief period of kination, the field crosses an enhanced symmetry point (ESP) at $\phi_{\mathrm{ESP}}$ and, due to its coupling to the $\chi$ field, nonperturbative

\footnotetext{
${ }^{2}$ We consider only $\phi>0$.
}

effects will generate a sea of $\chi$ particles. The energy budget for the particle production comes from the inflaton's kinetic energy and as such the particle production promptly traps the inflaton at $\phi_{\mathrm{ESP}}$. If $\chi$ is coupled to the standard model, its subsequent decays reheat the Universe. At this point the inflaton field's mass is primarily dependent on $\chi$, which until this point has been held at $\chi=0$. At a particular symmetry breaking scale (given by $f$ ) $\chi$ moves to its nonzero vacuum expectation value (VEV), providing the inflaton with a huge mass which acts to stop its motion. As such the $\varphi$ field is trapped at the ESP until late times when the residual energy density can act as dark energy. We consider $\chi$ to be the radial component of a complex field (the Peccei-Quinn field), whose angular degree of freedom is an axionlike particle (ALP) (it can be the QCD axion itself) which, whilst oscillating in the minimum of its potential, can describe the dark matter in the Universe.

\section{INFLATION}

The contribution to the inflaton potential from the coupling to the $\chi$ field will not affect the inflationary dynamics because $\chi=0$ during inflation, due to the large mass it obtains from the interaction term:

$$
m_{\chi}^{2}(\varphi)=g^{2} \phi_{\mathrm{ESP}}^{2}\left(e^{\frac{\varphi-\varphi_{\mathrm{ESP}}}{\sqrt{\alpha m_{\mathrm{Pl}}}}}-1\right)^{2},
$$

where $\varphi \rightarrow-\infty$ during inflation. Hence, we have the inflaton potential:

$$
V(\varphi)=V_{0} \exp \left(-\kappa e^{\frac{\varphi}{\sqrt{\alpha m_{\mathrm{P} 1}}}}\right) .
$$

From the expression above it is easy to compute the slowroll parameters, which are given by

$$
\begin{gathered}
\epsilon \equiv \frac{m_{\mathrm{Pl}}^{2}}{2}\left(\frac{V_{\varphi}}{V}\right)^{2}=\frac{\kappa^{2}}{2 \alpha} e^{\frac{2 \varphi}{\sqrt{\alpha m_{\mathrm{Pl}}}}}, \\
\eta \equiv \frac{m_{\mathrm{Pl}}^{2} V_{\varphi \varphi}}{V}=\frac{\kappa}{\alpha} e^{\frac{\varphi}{\sqrt{\alpha m_{\mathrm{Pl}}}}}\left(\kappa e^{\frac{\varphi}{\sqrt{\alpha m_{\mathrm{Pl}}}}}-1\right) .
\end{gathered}
$$

Defining the end of inflation as $\epsilon=1$ leads us to

$$
\varphi_{\mathrm{end}}=m_{\mathrm{Pl}} \sqrt{\alpha} \ln \frac{\sqrt{2 \alpha}}{\kappa},
$$

and

$$
\dot{\varphi}_{\mathrm{end}} \simeq \sqrt{\frac{2}{3} V_{\mathrm{end}}}
$$

where the latter was estimated by using the slow-roll equation $3 H \dot{\varphi} \simeq-V_{\varphi}$ and

$$
V_{\text {end }} \equiv V\left(\varphi_{\text {end }}\right)=V_{0} \mathrm{e}^{-\sqrt{2 \alpha}} .
$$


We can use $\varphi_{\text {end }}$ to find $\varphi$ when observable scales first left the horizon ${ }^{3}$

$$
\varphi_{*}=-\sqrt{\alpha} \ln \left[\frac{\kappa}{\alpha}\left(N_{*}+\sqrt{\frac{\alpha}{2}}\right)\right] m_{\mathrm{Pl}},
$$

where $N_{*}$ is the number of e-folds of inflation since the pivot scale exits the horizon. This gives us an idea of the value of the slow-roll parameters as a function of $N_{*}$

$$
\begin{gathered}
\epsilon_{*}=\frac{\alpha / 2}{\left(N_{*}+\sqrt{\alpha / 2}\right)^{2}} \simeq \frac{\alpha}{2 N_{*}^{2}}, \\
\eta_{*}=\frac{\alpha-N_{*}-\sqrt{\alpha / 2}}{\left(N_{*}+\sqrt{\alpha / 2}\right)^{2}} \simeq \frac{\alpha}{N_{*}^{2}}-\frac{1}{N_{*}}
\end{gathered}
$$

which it is nice to note are independent of $\kappa$. The spectral index and tensor to scalar ratio are hence also independent of $\kappa$ and given by

$$
\begin{gathered}
n_{\mathrm{s}}=1+2 \eta_{*}-6 \epsilon_{*} \simeq 1-\frac{\alpha}{N_{*}^{2}}-\frac{2}{N_{*}}, \\
r=16 \epsilon_{*} \simeq \frac{8 \alpha}{N_{*}^{2}} .
\end{gathered}
$$

As we would expect for a plateau inflation model, they match the Planck results [16] exceptionally well for a range of parameter values. The limiting observational constraint is $n_{s}=0.968 \pm 0.006$ ( $2 \sigma$ result) and the upper bound on the tensor to scalar ratio $r<r_{\text {bound }}$, where $r_{\text {bound }}=0.06$ (at $2 \sigma$ confidence level).

Using Eqs. (17) and (18) we can compute the upper bound on $N_{*}$. Assuming $N_{*}>31$, we find

$$
N_{*}<\frac{16}{8\left(1-n_{\mathrm{s}}\right)-r_{\text {bound }}} .
$$

Moreover, using Eq. (17) we can relate $\alpha$ to the number of e-folds $N_{*}$ :

\footnotetext{
${ }^{3} \mathrm{We}$ can also use this result to compute the field excursion during inflation. For the noncanonical field we find$$
\frac{\phi_{\mathrm{end}}-\phi_{*}}{m_{\mathrm{Pl}}}=\frac{\sqrt{2 \alpha}}{\kappa} \frac{N_{*}}{N_{*}+\sqrt{\frac{\alpha}{2}}} \simeq \frac{\sqrt{2 \alpha}}{\kappa} \ll 1,
$$

which is well bellow the Planck range. Switching to the canonical field we get

$$
\varphi-\varphi_{\text {end }}=-\sqrt{\alpha} m_{P} \ln \left(1+\sqrt{\frac{2}{\alpha}} N_{*}\right) \Rightarrow \Delta \varphi \simeq \sqrt{\alpha} m_{P},
$$

which is Planckian. Thus, we expect $r \sim 0.01$ from the Lyth bound.
}

$$
\alpha=\left(1-n_{\mathrm{s}}\right) N_{*}^{2}-2 N_{*} .
$$

Constraining the maximum possible value ${ }^{4}$ of $N_{*}<70$ and $\alpha \geq 1$ we find the allowed range for $N_{*}$ and $\alpha$ which is compatible with $\mathrm{CMB}$ observations to be

$$
\begin{gathered}
53 \leq N_{*}<70, \\
1 \leq \alpha<37 .
\end{gathered}
$$

Note that the allowed parameter range above depends sensitively on the value of $n_{\mathrm{s}}$. The above region is the maximum region within the $2 \sigma$ range of $n_{\mathrm{s}}$. For comparison, fixing $n_{\mathrm{s}}$ at the best fit value $n_{s}=0.968$ gives $63 \leq N_{*}<70$ and $1 \leq \alpha<15.8$.

The normalization of the power spectrum further constrains the model. The amplitude of the scalar spectrum at the pivot scale is given by

$$
A_{\mathrm{s}}=\frac{1}{24 \pi^{2} m_{\mathrm{Pl}}^{4}} \frac{V}{\epsilon} .
$$

Plugging Eqs. (8), (14) and (15) into the expression above we find

$$
\frac{V_{0}}{m_{\mathrm{Pl}}^{4}}=\frac{12 \pi^{2}}{\mathrm{e}^{2}}\left[\left(1-n_{\mathrm{s}}\right)-\frac{2}{N_{*}}\right] \mathrm{e}^{\left(1-n_{\mathrm{s}}\right) N_{*}} A_{\mathrm{s}},
$$

where we used Eq. (20). This expression relates the energy scale of inflation to the number of e-folds $N_{*}$. The Planck collaboration reports the value of $A_{\mathrm{s}}$ as [16]

$$
\ln \left(10^{10} A_{\mathrm{s}}\right)=3.094 \pm 0.034
$$

(at the $1 \sigma$ confidence level). On the upper panel of Fig. 1 we display the allowed range of $V_{0}$ for the best fit value of $A_{\mathrm{s}}$.

\footnotetext{
${ }^{4}$ We can estimate this as follows. The value of $N_{*}$ is increased by considering that, after inflation there is an "stiff" epoch when the barotropic parameter of the Universe is $\frac{1}{3}<w \leq 1$. The upper bound ensures that the speed of sound is not superluminal. The larger $w$ is and the longer this stiff period lasts, the more $N_{*}$ becomes. Thus, we can take $w=1$ as in kination, and we can assume that kination starts immediately after the end of inflation and until reheating. Then we have

$$
N_{*}=57+\frac{1}{3} \ln \left(\frac{V_{\mathrm{end}}^{1 / 4}}{T_{\text {reh }}}\right),
$$

where $T_{\text {reh }}$ is the reheating temperature. The observational bound on the inflation scale is $V_{\text {end }}^{1 / 4} \lesssim 10^{16} \mathrm{GeV}$. Saturating this bound, while considering the lowest $T_{\text {reh }}$ possible $\left(T_{\text {reh }} \gtrsim 10 \mathrm{MeV}\right.$, to avoid spoiling big bang nucleosynthesis) we find $N_{*}^{\max } \simeq 70$.
} 

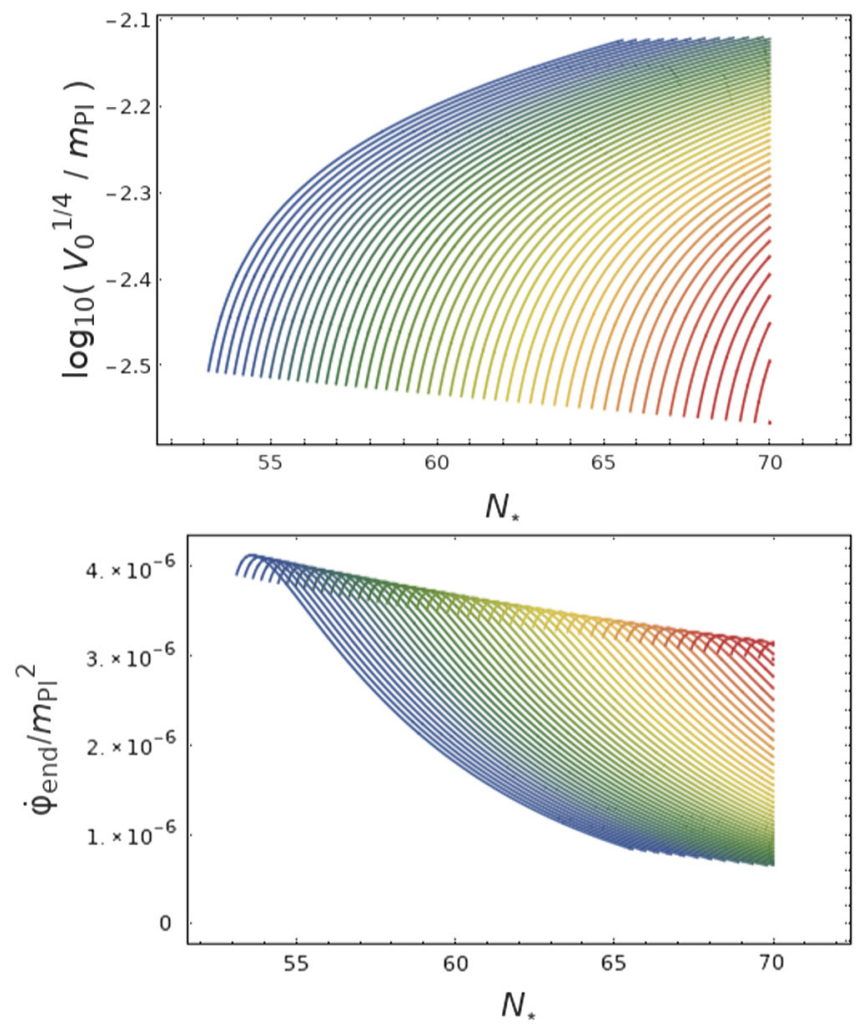

FIG. 1. The allowed range of $V_{0}$ values (upper) and the field velocity at the end of inflation $\dot{\varphi}_{\text {end }}$ (lower) as a function of $N_{*}$. Each curve represents a fixed value of the scalar spectral index $n_{\mathrm{s}}$. The blue leftmost curve is for the $2 \sigma$ lower bound $n_{s}=$ 0.962 and the red rightmost curve is for $n_{\mathrm{s}}=0.971$. We used the central value $\ln \left(10^{10} A_{\mathrm{s}}\right)=3.094$ of the spectrum normalization in Eq. (25).

Using $A_{\mathrm{s}}$ in Eq. (25) and plugging Eq. (24) into (12) we can find $\dot{\varphi}_{\text {end }}$. For the best fit value of $A_{\mathrm{s}}$ the allowed range of $\dot{\varphi}_{\text {end }}$ is show on the lower panel of Fig. 1. We see that the range is

$$
0.6<\frac{\dot{\varphi}_{\text {end }}}{m_{\mathrm{Pl}}^{2}} \times 10^{6}<4.2
$$

The $\kappa$ parameter in Eq. (1) determines the ratio of inflation energy density to the vacuum energy density. ${ }^{5}$ Plugging $\phi=\phi_{\mathrm{ESP}}$ and $\chi=f$ into that expression gives

\footnotetext{
${ }^{5}$ As we have shown in footnote 3 , for the noncanonical field during inflation we have that $\phi \sim \sqrt{\alpha} m_{P} / \kappa$. Thus, for the mass of the $\chi$-field during inflation from Eq. (1) we find $m_{\chi} \sim$ $(g \sqrt{\alpha} / \kappa) m_{P}$. Because $\alpha, g \sim 1$ and $\kappa \sim 100$ we have that the mass of the $\chi$-field during inflation is $m_{\chi} \sim 10^{-2} m_{P}$, i.e., comparable to the scale of grand unification and bigger that the Hubble scale during inflation. This means that the $\chi$-field is heavy during inflation, as we have assumed. In contrast, the inflaton field is light because $\eta$, calculated in Eq. (10), is much smaller than unity and this is why the value of the spectral index is close to unity.
}

$$
\kappa=\frac{m_{\mathrm{Pl}}}{\phi_{\mathrm{ESP}}} \ln \frac{V_{0}}{V_{\mathrm{vac}}}
$$

where $V_{\text {vac }}=V\left(\phi=\phi_{\mathrm{ESP}}, \chi=f\right) \simeq 10^{-12} \mathrm{eV}^{4} \simeq 10^{-120} m_{\mathrm{Pl}}^{4}$ is the vacuum energy density. Taking $V_{0}^{1 / 4}<10^{-2} m_{\mathrm{Pl}}$ (see Fig. 1) we find

$$
\kappa \frac{\phi_{\mathrm{ESP}}}{m_{\mathrm{Pl}}}<261
$$

\section{KINATION}

Once inflation ends, almost immediately the period of kination sets in. ${ }^{6}$ During kination the field is oblivious of the potential and the Klein-Gordon (KG) equation takes the form

$$
\ddot{\varphi}+3 H \dot{\varphi} \simeq 0,
$$

and the Friedmann equation is

$$
3 m_{\mathrm{Pl}}^{2} H^{2}=\frac{\dot{\varphi}^{2}}{2} .
$$

Substituting Eq. (30) into Eq. (29) gives

$$
\ddot{\varphi}+\sqrt{\frac{3}{2}} \frac{\dot{\varphi}^{2}}{m_{\mathrm{Pl}}}=0 .
$$

Integrating the above equation, we find the solutions

$$
\begin{gathered}
\varphi=\varphi_{0}+\sqrt{\frac{2}{3}} m_{\mathrm{Pl}} \ln \left[1+\sqrt{\frac{3}{2}} \frac{\dot{\varphi}_{0}}{m_{\mathrm{Pl}}}\left(t-t_{0}\right)\right], \\
\dot{\varphi}=\dot{\varphi}_{0} \exp \left[-\sqrt{\frac{3}{2}} \frac{\left(\varphi-\varphi_{0}\right)}{m_{\mathrm{Pl}}}\right] .
\end{gathered}
$$

where the subscript " 0 " refers to the initial value in the integration. In our case $\varphi_{0} \simeq \varphi_{\text {end }}$ and $\dot{\varphi}_{0} \simeq \dot{\varphi}_{\text {end }}$ in Eqs. (11) and (12) respectively, meaning the above equations become

$\varphi_{\text {kin }}=m_{\mathrm{Pl}} \sqrt{\alpha} \ln \frac{\sqrt{2 \alpha}}{\kappa}+\sqrt{\frac{2}{3}} m_{\mathrm{Pl}} \ln \left[1+\frac{\sqrt{V_{\mathrm{end}}}}{m_{\mathrm{Pl}}}\left(t-t_{0}\right)\right]$,

$$
\dot{\varphi}_{\text {kin }}=\sqrt{\frac{2}{3} V_{\text {end }}}\left(\frac{\sqrt{2 \alpha}}{\kappa}\right)^{\sqrt{\frac{3 \alpha}{2}}} \mathrm{e}^{-\sqrt{\frac{3}{2 m_{\mathrm{P}}}}} .
$$

Taking $\varphi_{0} \simeq \varphi_{\text {end }}$ and $\dot{\varphi}_{0} \simeq \dot{\varphi}_{\text {end }}$ presumes an immediate transition from inflation to kination, when $\epsilon=1$.

\footnotetext{
${ }^{6} \mathrm{We}$ have confirmed this using numerical simulations.
} 


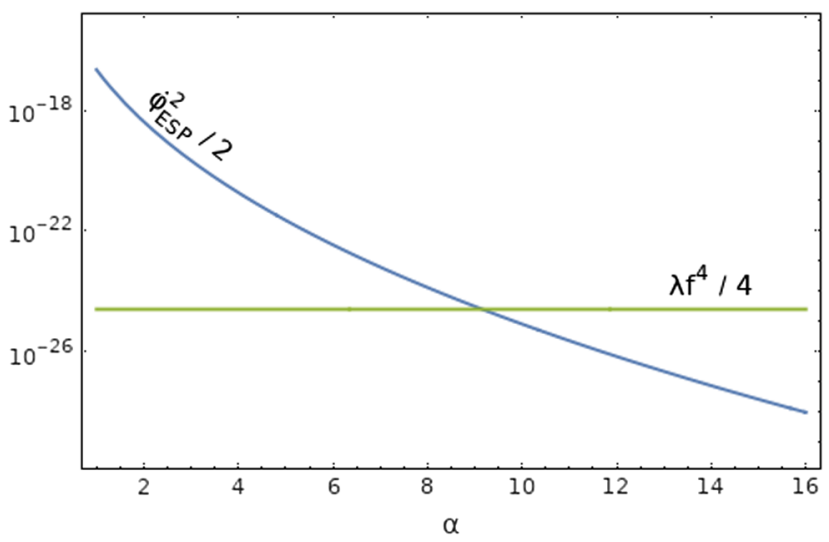

FIG. 2. The inflaton kinetic energy density at ESP (blue curve) and the false vacuum energy density (green line) as a function of $\alpha$. In this plot we used the best fit value of $n_{\mathrm{s}}$ (within $2 \sigma$ range the result depends mildly on the precise value of $n_{\mathrm{s}}$ ) and we took $\lambda=1$ and $f=10^{12} \mathrm{GeV}$.

During kination, the kinetic energy density of the inflaton scales as $\dot{\varphi}^{2} / 2 \propto a^{-6}$, where $a$ is the scale factor. Therefore the false vacuum energy density $\lambda f^{4} / 4$ might come to dominate at some later times. For the sake of simplicity we restrict the model to the parameter range where this never happens until $\varphi$ reaches ESP.

To find this regime we can compute $\dot{\varphi}_{\text {ESP }}$ using Eq. (35). Plugging Eqs. (5) and (13) into the latter we obtain

$$
\dot{\varphi}_{\mathrm{ESP}}=\frac{\sqrt{2 V_{0}(\alpha) / 3}}{\mathrm{e}^{\sqrt{\alpha / 2}}}\left(\frac{\sqrt{2 \alpha} m_{\mathrm{PI}}}{\kappa(\alpha) \phi_{\mathrm{ESP}}}\right)^{\sqrt{\frac{3 \alpha}{2}}},
$$

where the argument in $V_{0}(\alpha)$ and $\kappa(\alpha)$ is to remind us that observations constrain $\kappa$ and $V_{0}$ to be functions of $\alpha$ via Eqs. (27), (24) and (20).

Using Eq. (36), we plot $\dot{\varphi}_{\mathrm{ESP}} / 2$ as a function of $\alpha$ in Fig. 2 along with the constant value of $\lambda f^{4} / 4$. As we can easily see the universe is in the regime of kination at the ESP if

$$
\frac{\lambda f^{4}}{2 \dot{\varphi}_{\mathrm{ESP}}^{2}}<1 \Rightarrow \alpha<10
$$

The precise value of course depends on $f$ and (only mildly) on $n_{\mathrm{s}}$, but we adopt this bound as our reference value.

\section{INFLATON TRAPPING AT THE ESP}

During the period of kination, the inflaton follows the equation of motion (29). Once it approaches $\phi \rightarrow \phi_{\mathrm{ESP}}$, the tachyonic and resonant excitations of the $\chi$ field produce large numbers of particles. These particles backreact onto the motion of the inflaton, creating an effective linear potential for the latter. If the production of $\chi$ particles is efficient, then the inflaton's fast rolling is halted by trapping it at the ESP.
As we are going to see, the trapping can be very abrupt. Therefore, it is a good approximation to neglect the expansion of the universe. Moreover, as we will show a posteriori, $\varphi$ oscillates around the ESP with an amplitude much smaller than $m_{\mathrm{Pl}},\left|\varphi-\varphi_{\mathrm{ESP}}\right| \ll m_{\mathrm{Pl}}$. Therefore, in considerations of the trapping process, it is enough to study the Lagrangian up to the first order in $\left|\varphi-\varphi_{\mathrm{ESP}}\right| / m_{\mathrm{Pl}}$. In this case, we find from Eq. (6)

$V \simeq V_{\mathrm{vac}}\left(\frac{V_{\mathrm{vac}}}{V_{0}}\right)^{\frac{\varphi-\varphi_{\mathrm{ESP}}}{\sqrt{\alpha m_{\mathrm{Pl}}}}}+\frac{1}{2} \gamma^{2}\left(\varphi-\varphi_{\mathrm{ESP}}\right)^{2} \chi^{2}+V(\chi)$,

where

$$
\gamma^{2} \equiv \frac{g^{2} \phi_{\mathrm{ESP}}^{2}}{\alpha m_{\mathrm{Pl}}^{2}}
$$

is the effective quartic coupling constant close to the ESP. The first term in Eq. (38) sets the scale of the vacuum energy. It is much smaller than $\lambda f^{4} / 4$ and can be neglected during the inflaton trapping phase.

With the above assumptions in mind we can write the equation of motion for the inflaton as

$$
\ddot{\varphi}+\gamma^{2}\left\langle\chi^{2}\right\rangle\left(\varphi-\varphi_{\mathrm{ESP}}\right) \simeq 0 .
$$

Initially the expectation value of the $\chi$ field is zero, which makes $\varphi$ massless at the classical level. However, quantum corrections due to the interaction term generate an effective mass for the $\varphi$ field. At the first order such corrections can be accounted for by using the Hartree approximation, which has been employed in the expression above.

The trapping of the inflaton $\varphi$ by the resonant production of $\chi$ particles has been studied in great detail in Ref. [27]. In that work, the primary source of particle production is the parametric resonance. In our case, $\omega_{k}^{2}$ (defined below) is not positive definite, therefore the $\chi$ field is also excited by the tachyonic instability close to the ESP. Moreover, in our model the quartic self-interaction of the $\chi$ field can affect the particle production too. To investigate these processes we start by writing the equation of motion for the mode functions of the $\chi$ field:

$$
\ddot{\chi}_{k}+\omega_{k}^{2} \chi_{k}=0
$$

where

$$
\omega_{k}^{2}=k^{2}+\gamma^{2}\left(\varphi-\varphi_{\mathrm{ESP}}\right)^{2}+\lambda\left(3\left\langle\chi^{2}\right\rangle-f^{2}\right) .
$$

Initially $\left\langle\chi^{2}\right\rangle$ is negligible and we can clearly see in the above equation that $\chi_{k}$ modes with $k<k_{\mathrm{c}}$ acquire an effective imaginary mass as $\varphi \rightarrow \varphi_{\mathrm{ESP}}$, where $k_{\mathrm{c}}$ is the critical wave number

$$
k_{\mathrm{c}} \equiv \sqrt{\lambda} f .
$$


These modes are unstable and start growing due to the tachyonic instability. The process is similar to the one analyzed in Ref. [36]. To compute the production of $\chi$ particles note, first, that in a narrow window

$$
\left|\varphi-\varphi_{\mathrm{ESP}}\right| \leq \frac{\sqrt{\lambda} f}{\gamma}
$$

we can approximate the evolution of $\varphi$ linearly [see Eq. (32)]

$$
\varphi-\varphi_{\mathrm{ESP}} \simeq \dot{\varphi}_{\mathrm{ESP}} \tau
$$

where $\tau \equiv t-t_{\mathrm{ESP}}$ and $t_{\mathrm{ESP}}$ is defined as $\varphi\left(t_{\mathrm{ESP}}\right) \equiv \varphi_{\mathrm{ESP}}$ and $\dot{\varphi}_{\text {ESP }}$ in the above equation is the field velocity at the ESP from Eq. (36).

Using Eq. (45) we find that after the first crossing of the ESP the occupation number of $\chi_{k}$ mode is given by [36]

$$
n_{k}=\exp \int_{\tau_{-}}^{\tau_{+}} 2 \sqrt{-\omega^{2}\left(\tau^{\prime}\right)} \mathrm{d} \tau^{\prime}
$$

and $\omega^{2}\left(\tau_{ \pm}\right)=0$, that is

$$
\tau_{ \pm}= \pm \sqrt{\frac{\lambda f^{2}-k^{2}}{\gamma^{2} \dot{\varphi}_{\mathrm{ESP}}^{2}}} .
$$

It is easy to integrate Eq. (46), which gives

$$
n_{k}=\mathrm{e}^{\pi \frac{\lambda f^{2}-k^{2}}{\gamma \dot{\varphi}_{\mathrm{ESP}}}}
$$

Integrating over all $k$ wave numbers, we obtain the total occupation number of newly produced particles after the first passage of the ESP

$$
\begin{aligned}
n_{\chi 1} & \simeq \frac{1}{2 \pi^{2}} \int_{0}^{\infty} n_{k} k^{2} \mathrm{~d} k \\
& \simeq \frac{\left(\gamma \dot{\varphi}_{\mathrm{ESP}}\right)^{3 / 2}}{(2 \pi)^{3}}\left[-2 s^{1 / 4}+\mathrm{e}^{\pi s^{1 / 2}} \operatorname{Erf}\left(\sqrt{\pi} s^{1 / 4}\right)\right],
\end{aligned}
$$

where $\operatorname{Erf}(x) \equiv 2 / \sqrt{\pi} \int_{0}^{z} \mathrm{e}^{-t^{2}} \mathrm{~d} t$ is the error function and

$$
s \equiv\left(\frac{\lambda f^{2}}{\gamma \dot{\varphi}_{\mathrm{ESP}}}+\frac{2}{3^{3 / 2}}\right)^{2} .
$$

The first term in the parenthesis is positive. Therefore, $s^{1 / 4} \geq \sqrt{2} / 3^{3 / 4} \quad$ and $\quad \operatorname{Erf}\left(\sqrt{\pi} s^{1 / 4}\right) \simeq 1$. Moreover, $\exp \{\pi \sqrt{s}\}>s^{1 / 4}$ and we can write

$$
n_{\chi 1} \simeq \frac{\left(\gamma \dot{\varphi}_{\mathrm{ESP}}\right)^{3 / 2}}{(2 \pi)^{3}} \mathrm{e}^{\pi\left(\frac{\lambda f^{2}}{\gamma \dot{\mathrm{ESP}}^{2}}+\frac{2}{3^{3 / 2}}\right)}
$$

Similarly we can compute the dispersion $\left\langle\chi^{2}\right\rangle$ [37]

$$
\begin{aligned}
\left\langle\chi^{2}\right\rangle & =\frac{1}{2 \pi^{2}} \int \frac{n_{k} k^{2}}{\omega_{k}} \mathrm{~d} k \\
& \simeq \frac{n_{\chi}}{\gamma\left|\varphi-\varphi_{\mathrm{ESP}}\right|},
\end{aligned}
$$

where we used the fact that $\gamma\left(\varphi-\varphi_{\mathrm{ESP}}\right)>k_{\mathrm{c}}$ in the nontachyonic regime.

Plugging Eq. (54) into (40), we can write the inflaton equation of motion as

$$
\ddot{\varphi}+\gamma n_{\chi} \operatorname{sign}\left(\varphi-\varphi_{\mathrm{ESP}}\right) \simeq 0,
$$

where $\operatorname{sign}\left(\varphi-\varphi_{\mathrm{ESP}}\right)$ is the signature of $\varphi-\varphi_{\mathrm{ESP}}$. This equation describes oscillations in a linear potential: at the time

$$
\tau_{1} \equiv \frac{\dot{\varphi}_{\mathrm{ESP}}}{\gamma n_{\chi 1}}
$$

the field $\varphi$ reaches the value $\varphi_{1}=\varphi_{\mathrm{ESP}}+\Phi_{1}$ and rolls back toward $\varphi_{\mathrm{ESP}}$, where

$$
\Phi_{1} \simeq \frac{1}{2} \frac{\dot{\varphi}_{\mathrm{ESP}}^{2}}{\gamma n_{\chi 1}}
$$

is the amplitude of the first oscillation.

Up to now we have assumed that the expansion of the universe does not affect the trapping process. This is justified if the timescale $\tau_{1} \simeq 2\left(\varphi_{1}-\varphi_{\mathrm{ESP}}\right) / \dot{\varphi}_{\mathrm{ESP}}$ is much shorter than the Hubble time $H_{\mathrm{ESP}}^{-1}$ at the ESP; that is $H_{\mathrm{ESP}} \ll \dot{\varphi}_{\mathrm{ESP}} / 2 \Phi_{1}$. Using $H_{\mathrm{ESP}} \simeq \dot{\varphi}_{\mathrm{ESP}} / \sqrt{6} m_{\mathrm{Pl}}$, in agreement with our assumption $\alpha<10$ (see Fig. 2), we find that this is the case when

$$
\Phi_{1} \ll \sqrt{\frac{3}{2}} m_{\mathrm{Pl}} .
$$

Plugging Eqs. (36) and (52) into Eq. (57), we find

$$
\Phi_{1} \simeq \frac{4 \pi^{3}}{\gamma^{5 / 2}} \frac{\left[\frac{2}{3} V_{0}(\alpha)\right]^{1 / 4}}{\mathrm{e}^{\pi\left(\frac{\lambda f^{2}}{\gamma \dot{\mathrm{ESP}}}+\frac{2}{3^{3 / 2}}\right)}}\left(\frac{\sqrt{2 \alpha}}{\mathrm{e}^{1 / \sqrt{3}}} \frac{m_{\mathrm{Pl}}}{\kappa(\alpha) \phi_{\mathrm{ESP}}}\right)^{\frac{1}{2} \sqrt{\frac{3 \alpha}{2}}} .
$$

To find the range where Eq. (58) is satisfied we plot $\log _{10} \Phi_{1}$ as a function of $\alpha$ and $\gamma$ in Fig. 3. We can see that Eq. (58) can be easily satisfied for reasonable values of $\gamma$.

Each time $\varphi$ crosses the ESP, the $\chi$ field experiences a burst of particle production. New particles strengthen the backreaction onto the motion of $\varphi$, causing an exponential decay of the oscillation amplitude. Via the quartic selfinteraction term these particles also generate a contribution to the effective mass of the $\chi$ field. At the level of the 


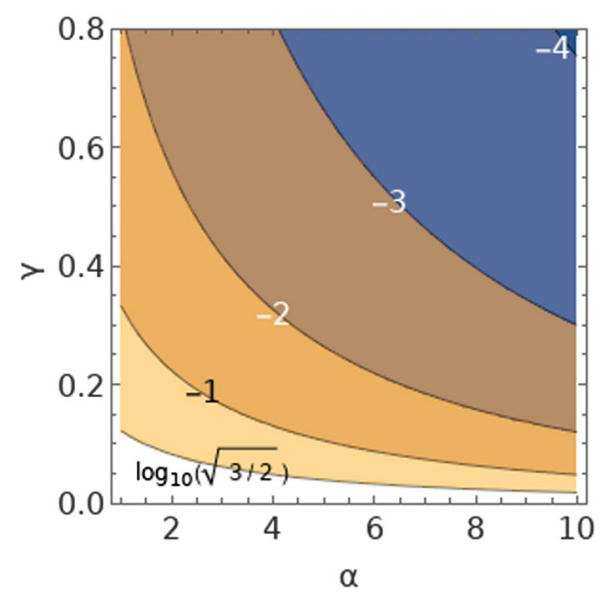

FIG. 3. A contour plot of $\log _{10}\left(\Phi_{1} / m_{\mathrm{Pl}}\right)$ values as a function of $\alpha$ and $\gamma$ [see Eq. (59)]. In this plot we used $\lambda f^{2} / \gamma \dot{\varphi}_{\text {ESP }} \ll 2 / 3^{3 / 2}$ and the best fit value $n_{\mathrm{s}}=0.968$. The plot changes insignificantly within the allowed $2 \sigma$ range of $n_{\mathrm{s}}$. The white region is where the condition in Eq. (58) is violated, i.e., where the expansion of the Universe cannot be neglected.

Hartree approximation these interactions are taken care of by the $3 \lambda\left\langle\chi^{2}\right\rangle$ term in Eq. (42). The tachyonic particle production is effective as long as the $3 \lambda\left\langle\chi^{2}\right\rangle$ term is smaller than $\lambda f^{2}$.

To compute the occupation number at the end of the tachyonic instability we first find the minimum of $\omega_{k=0}^{2}$ in Eq. (42) which is located at $\left[\gamma\left(\varphi_{\min }-\varphi_{\mathrm{ESP}}\right)\right]^{3}=3 \lambda n_{\chi} / 2$, where we used Eq. (54). Plugging this back into the expression of $\omega_{k=0}^{2}$ and equating it to zero, we find that the tachyonic resonance stops at

$$
n_{\chi, \text { tach }} \simeq \frac{2}{3^{5 / 2}} \sqrt{\lambda} f^{3}
$$

At this moment the oscillation amplitude is given by

$$
\Phi_{\mathrm{tach}} \simeq \frac{3^{5 / 2}}{4} \frac{\dot{\varphi}_{\mathrm{ESP}}^{2}}{\gamma \sqrt{\lambda} f^{3}} .
$$

We can compare $n_{\chi \text {,tach }}$ with the number density produced after the first oscillation in Eq. (52)

$$
\frac{n_{\chi 1}}{n_{\chi, \text { tach }}} \simeq \frac{3^{5 / 2}}{16 \pi^{3}} \lambda\left(\frac{\gamma \dot{\varphi}_{\text {ESP }}}{\lambda f^{2}}\right)^{3 / 2} \mathrm{e}^{\pi\left(\frac{\lambda f^{2}}{\gamma \varphi_{\mathrm{ESP}}}+\frac{2}{3^{3 / 2}}\right)}
$$

For $\gamma \dot{\varphi}_{\mathrm{ESP}} / \lambda f^{2}=2 \pi / 3$ the right-hand side of the above expression is minimal and given by

$$
\left.\frac{n_{\chi 1}}{n_{\chi, \text { tach }}}\right|_{\text {min }} \sim \lambda \lesssim 1 .
$$

Thus, depending on the magnitude of $\gamma \dot{\varphi}_{\mathrm{ESP}} / \lambda f^{2}$, it might take several passages through the ESP before the tachyonic resonance is terminated.
The end of the tachyonic resonance does not necessarily imply the end of particle production though. After $\omega_{k}^{2}$ becomes positive definite for all values of $k$, particles may still be produced by the parametric resonance. Such a production continues as long as the adiabaticity condition

$$
\frac{\left|\dot{\omega}_{k}\right|}{\omega_{k}^{2}}<1
$$

is broken within some range of $\varphi$ values $\Delta \varphi_{\text {nad }}$, that is $\left|\dot{\omega}_{k}\right| / \omega_{k}^{2} \geq 1$ for $\varphi-\varphi_{\text {ESP }} \in\left[-\Delta \varphi_{\text {nad }}, \Delta \varphi_{\text {nad }}\right] / 2$. As $\left\langle\chi^{2}\right\rangle$ continues to grow with each burst of particle production, it will eventually shut down the parametric resonance too.

The shut-down is caused by one of the two effects, whichever happens first: either the oscillation amplitude $\Phi$ becomes smaller than $\Delta \varphi_{\text {nad }}$ [27] (Case 1) or quartic selfinteractions of the $\chi$ field render it too heavy to be excited (Case 2). The choice between the two cases is determined by the magnitude of the ratio $\lambda / \gamma^{2}$.

Let us consider these two possibilities in turn. To do that we can safely employ Eq. (45) within the narrow nonadiabaticity window. Hence we can write

$$
\frac{\dot{\omega}_{k}}{\omega_{k}^{2}} \simeq \frac{\gamma^{2}\left(\varphi-\varphi_{\mathrm{ESP}}\right) \dot{\varphi}_{\mathrm{ESP}}}{\left[k^{2}+\gamma^{2}\left(\varphi-\varphi_{\mathrm{ESP}}\right)^{2}+\lambda\left(3\left\langle\chi^{2}\right\rangle-f^{2}\right)\right]^{3 / 2}},
$$

where in the adiabatic regime $\left\langle\chi^{2}\right\rangle$ is given by the expression in Eq. (54).

In Case 1 the resonance stops before the $\lambda\left(3\left\langle\chi^{2}\right\rangle-f^{2}\right)$ term in Eq. (65) becomes important and we neglect it. This gives $\Delta \varphi_{\text {nad }} \simeq\left(\dot{\varphi}_{\mathrm{ESP}} / \gamma\right)^{1 / 2}$. Once the oscillation amplitude $\Phi=\dot{\varphi}_{\mathrm{ESP}}^{2} / 2 \gamma n_{\chi}$ drops below this value, particles no longer grow via the process of parametric resonance. Equating $\Delta \varphi_{\text {nad }}=\Phi$ we find

$$
\Phi_{\text {fin } 1} \simeq\left(\frac{\dot{\varphi}_{\text {ESP }}}{\gamma}\right)^{1 / 2}
$$

and

$$
n_{\chi, \mathrm{fin} 1} \simeq \frac{1}{2} \frac{\dot{\varphi}_{\mathrm{ESP}}^{3 / 2}}{\gamma^{1 / 2}}
$$

where the subscript "fin1" signifies the case where the resonance stops because the inflaton oscillation amplitude drops bellow $\Delta \varphi_{\text {nad }}$. We can compute the energy density $\rho_{\chi \text { fin } 1} \simeq \gamma \Phi_{\text {fin } 1} n_{\chi, \text { fin } 1}$ in the $\chi$ particles at that moment

$$
\rho_{\chi \mathrm{fin} 1} \simeq \frac{1}{2} \dot{\varphi}_{\mathrm{ESP}}^{2},
$$

which is about the same as the initial kinetic energy density of the inflaton when it first crosses the ESP.

In Case 1 we could neglect $\chi$ field self-interactions in Eq. (65). This rendered $\Delta \varphi_{\text {nad }} \simeq$ constant. On the other 
hand, if $\lambda / \gamma^{2}$ is large, which corresponds to Case 2, such self-interactions cannot be neglected and the $\left\langle\chi^{2}\right\rangle$ term in Eq. (65) becomes significant. As the importance of this term grows, the nonadiabaticity region $\Delta \varphi_{\text {nad }}$ shrinks to zero eventually halting the resonance.

To estimate the end of the resonance we find the moment when the maximum value of the ratio in Eq. (65) becomes smaller than one. For the $k=0$ mode the maximum value of this ratio is approximately

$$
\left.\frac{\dot{\omega}_{k=0}}{\omega_{k=0}^{2}}\right|_{\max } \simeq \frac{\gamma \dot{\varphi}_{\mathrm{ESP}}}{6\left(\lambda n_{\chi}\right)^{2 / 3}}
$$

where we used $3\left\langle\chi^{2}\right\rangle>f^{2}$ and Eq. (54). The resonance becomes inefficient once this value falls bellow unity. Hence, we can consider the particle production to be over when

$$
n_{\chi, \mathrm{fin} 2} \simeq \frac{\left(\gamma \dot{\varphi}_{\mathrm{ESP}} / 6\right)^{3 / 2}}{\lambda}
$$

Plugging this value into Eq. (55) we find that the inflaton oscillation amplitude at this point is

$$
\Phi_{\mathrm{fin} 2} \simeq 7 \frac{\lambda}{\gamma^{2}}\left(\frac{\dot{\varphi}_{\mathrm{ESP}}}{\gamma}\right)^{1 / 2} .
$$

Comparing Eq. (67) with (70) and Eq. (66) with (71) we see that the first mechanism is responsible for the end of the resonance if

$$
\frac{\lambda}{\gamma^{2}}<\frac{1}{7}
$$

In this case the energy density of $\chi$ particles is comparable to the inflaton's initial kinetic energy (see Eq. (68). In the opposite regime, the strong quartic self-interaction $\lambda \chi^{4}$ shuts down the resonance much earlier, leaving a larger fraction of the energy budget in the inflaton sector. Moreover, the inflaton oscillation amplitude is larger too. In summary, a stronger $\chi$ field self-interaction results in less efficient inflaton trapping.

\section{REHEATING}

As we have shown, after crossing the ESP the total kinetic density of the inflaton decays into radiation through resonant production of $\chi$-particles. ${ }^{7}$ Thus we expect

\footnotetext{
${ }^{7}$ The case when the quartic self-interaction of the $\chi$-particles stops their resonant production early (Case 2, discussed above) introduces the extra complication of the perturbative decay of the oscillating inflaton condensate. For simplicity, we consider only Case 1, which amounts to satisfying the bound in Eq. (72).
}

$$
\frac{1}{2} \dot{\varphi}_{\mathrm{ESP}}^{2} \simeq \frac{\pi^{2}}{30} g_{*} T_{\mathrm{reh}}^{4}
$$

where $g_{*}=\mathcal{O}(100)$ is the effective relativistic degrees of freedom. Using the above and Eq. (36) we obtain

$$
T_{\text {reh }}=\left[\frac{10}{\pi^{2} g_{*}} V_{\text {end }}\left(\frac{\sqrt{2 \alpha}}{\kappa} \frac{m_{\mathrm{Pl}}}{\phi_{\mathrm{ESP}}}\right)^{\sqrt{6 \alpha}}\right]^{1 / 4},
$$

where we used $V_{\text {end }}=V_{0} e^{-\sqrt{2 \alpha}}$ according to Eq. (13). To get a feeling about the magnitude of $T_{\text {reh }}$ we take $\phi_{\mathrm{ESP}}=m_{\mathrm{Pl}}$ and $V_{\mathrm{end}}^{1 / 4} \sim V_{0}^{1 / 4} \sim 10^{-2} m_{\mathrm{Pl}}$, in which case we have

$$
T_{\text {reh }} \sim 10^{-3} m_{\mathrm{Pl}}\left(\frac{\sqrt{2 \alpha}}{\kappa}\right)^{\sqrt{3 \alpha / 8}} .
$$

As we show below $\kappa=\mathcal{O}(100)$ in order to have successful dark energy. Then $\alpha \in[1.5,10]$ gives $T_{\text {reh }} \sim 10^{11-13} \mathrm{GeV}$. This means that, if $T_{\text {reh }}$ is large, thermal corrections might restore the Peccei-Quinn symmetry, unless $\kappa$ is rather large, approaching the bound in Eq. (28). Actually, in this case, the Peccei-Quinn symmetry is thermally broken later on, after the onset of radiation domination. In contrast, if the reheating temperature is not very large, the Peccei-Quinn symmetry is broken once the inflaton rolls towards the VEV regardless of thermal corrections. In both cases, the axion does not exist during inflation and so there is no problem with axion isocurvature perturbations.

Knowing the reheating temperature in Eq. (74), we can calculate the number of e-folds of inflation from the recognisable equation

$$
\begin{aligned}
N_{*}= & 61.2+\frac{1}{3(1+w)} \ln \left(\frac{g_{*} \pi^{2}}{60}\right) \\
& +\frac{(3 w-1)}{3(1+w)} \ln \left(\frac{V_{\text {end }}^{1 / 4}}{T_{\text {reh }}}\right)+\ln \left(\frac{V_{\text {end }}^{1 / 4}}{m_{\mathrm{Pl}}}\right) .
\end{aligned}
$$

Presuming the kinetically dominated inflaton field is still the dominant component of the energy density until the produced radiation dominates, we have $w=1$ and the above becomes

$$
N_{*}=61.7+\frac{1}{3} \ln \left(\frac{V_{\mathrm{end}}^{1 / 4}}{T_{\mathrm{reh}}}\right)+\ln \left(\frac{V_{\mathrm{end}}^{1 / 4}}{m_{\mathrm{Pl}}}\right) .
$$

\section{RESULTS}

To find the energy scale at the end of inflation we can first substitute $n_{s}$ from Eq. (20) into Eq. (24) to obtain 


$$
\frac{V_{0}}{m_{\mathrm{Pl}}^{4}}=\frac{12 \pi^{2} \alpha A_{\mathrm{s}}}{N_{*}^{2}} e^{\alpha / N_{*}},
$$

which is independent of $\kappa$. Using that $\alpha<N_{*}$ and $A_{\mathrm{s}}=$ $(2.208 \pm 0.075) \times 10^{-9}$ [cf. Eq. (25)], this always gives

$$
V_{0}^{1 / 4} \simeq 2 \times 10^{-3} \alpha^{1 / 4} m_{\mathrm{Pl}} .
$$

Using this value to find $V_{\text {end }}=V_{0} e^{-\sqrt{2 \alpha}}$ we see

$$
V_{\mathrm{end}}^{1 / 4}=2 \times 10^{-3} \alpha^{1 / 4} e^{-\sqrt{\alpha / 8}} m_{\mathrm{Pl}} \sim 10^{-3} m_{\mathrm{Pl}},
$$

where $\alpha \in[1.5,10]$ (see also Fig. 1). This is close to the scale of a grand unified theory (GUT) as expected.

Rearranging Eq. (74) we readily obtain

$$
\frac{V_{\text {end }}^{1 / 4}}{T_{\text {reh }}}=\left[\frac{\pi^{2} g_{*}}{10}\left(\frac{\kappa}{\sqrt{2 \alpha}} \frac{\phi_{\mathrm{ESP}}}{m_{\mathrm{Pl}}}\right)^{\sqrt{6 \alpha}}\right]^{1 / 4} .
$$

Also, using $V_{\text {end }}=V_{0} e^{-\sqrt{2 \alpha}}$ and Eq. (78) we find

$$
\frac{V_{\mathrm{end}}^{1 / 4}}{m_{\mathrm{Pl}}}=\frac{\left(12 \pi^{2} \alpha A_{\mathrm{s}}\right)^{1 / 4}}{\sqrt{N}_{*}} \exp \left(\frac{\alpha}{4 N_{*}}-\sqrt{\frac{\alpha}{8}}\right) .
$$

Combining Eqs. (81) and (82) with Eq. (77) and after some algebra we end up with

$$
N_{*} \simeq 56.3+\sqrt{\frac{\alpha}{24}} \ln \left(\frac{\kappa}{\sqrt{2 \alpha}} \frac{\phi_{\mathrm{ESP}}}{m_{\mathrm{Pl}}}\right),
$$

where we took $\exp \left(\frac{\alpha}{4 N_{*}}-\sqrt{\frac{\alpha}{8}}\right) \sim 1$.

The value of $\kappa$ is determined by the necessity for the residual potential energy of $\varphi$ to act as dark energy at late times [see Eq. (27)]. As such

$$
V\left(\varphi_{\mathrm{ESP}}\right)=V_{0} \exp \left(-\kappa e^{\frac{\varphi_{\mathrm{ESP}}}{\sqrt{\alpha m_{\mathrm{Pl}}}}}\right) \simeq 10^{-120} m_{\mathrm{Pl}}^{4},
$$

which rearranges to

$$
\kappa \approx 244 e^{\frac{\varphi_{\mathrm{ESP}}}{\sqrt{\alpha} m_{\mathrm{Pl}}}}
$$

[cf. Eq. (28)] Using this, Eq. (83) is written as

$$
N_{*} \simeq 56.3+\sqrt{\frac{\alpha}{24}}\left[\ln \left(\frac{244}{\sqrt{2 \alpha}}\right)+\frac{2 \varphi_{\mathrm{ESP}}}{\sqrt{\alpha} m_{\mathrm{PI}}}\right],
$$

where we used $\ln \left(\frac{\phi_{\mathrm{ESP}}}{m_{\mathrm{Pl}}}\right)=\frac{\varphi_{\mathrm{ESP}}}{\sqrt{\alpha} m_{\mathrm{Pl}}}$, according to Eq. (5).

We know that the pole in the non-canonical field potential, $\phi=0$ is transposed to $\varphi=-\infty$ with our field redefinition, generating a plateau in the model which provides the slow-roll regime for inflation. The value of $\kappa$ effectively shifts the position of the edge of the plateau,
TABLE I. Inflationary observables taking $\varphi_{\mathrm{ESP}}=0$.

\begin{tabular}{lccc}
\hline \hline$\alpha$ & $N_{*}$ & $n_{s}$ & $r$ \\
\hline $3 / 2$ & 57.5 & 0.965 & 0.0024 \\
10 & 58.9 & 0.963 & 0.023 \\
\hline \hline
\end{tabular}

which explains why the value of $\varphi_{\mathrm{ESP}}$ differs for each $\kappa$ value in this equation.

It is straightforward to obtain the inflationary observables $n_{s}(\alpha)$ and $r(\alpha)$ using Eqs. (17) and (18) with Eq. (86). As an indicative choice we consider $\varphi_{\mathrm{ESP}}=0$. In this case, the results are shown in Table I and depicted in Fig. 4. As evident, there is excellent agreement with the Planck results [38].

Remarkably, the values of the inflationary observables do not change much when varying $\varphi_{\mathrm{ESP}}$. For example, taking $\varphi_{\mathrm{ESP}}=m_{\mathrm{Pl}}$ adds $2 / \sqrt{24} \simeq 0.4$ to the value of $N_{*}$, so that $N_{*}(3 / 2)=57.9\left(N_{*}(10)=59.3\right)$. Yet, the resulting values of $n_{s}$ and $r$ remain virtually unchanged, given by the same values shown in Table I. Thus, our results are robust and only very weakly dependent on the location of the ESP (value of $\varphi_{\mathrm{ESP}}$ ), which means that no tuning is required to match the observations.

This is less so with the value of $\kappa$. Indeed, from Eq. (85) we see that setting $\varphi_{\mathrm{ESP}}=0$ requires

$$
\kappa \approx 244
$$

to obtain the correct energy density for dark energy. If instead we have $\varphi_{\mathrm{ESP}}=m_{\mathrm{Pl}}$, with $\alpha=10$, we have

$$
\kappa \approx 335
$$

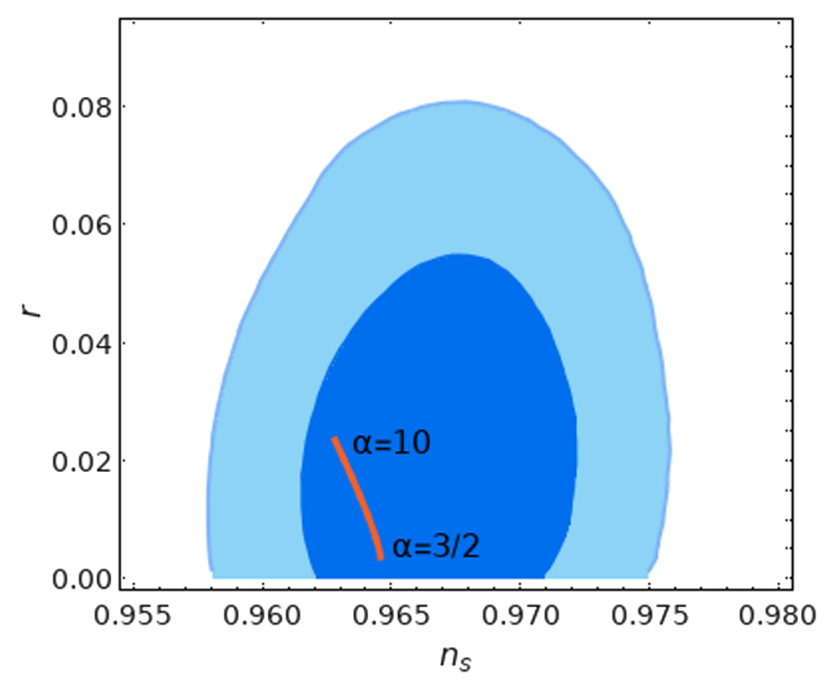

FIG. 4. The $1 \sigma$ and $2 \sigma$ contours of the Planck constraints from Ref. [38]. The red curve is the prediction of our model assuming Eq. (73). 
In all cases however, we see that $\kappa=\mathcal{O}(100)$, which means that the inflaton field in the exponent of the potential in Eq. (1) is suppressed by the GUT scale $m_{\mathrm{Pl}} / \kappa \sim 10^{16} \mathrm{GeV}$.

\section{CONCLUSIONS}

We have analyzed a new model of quintessential inflation, inspired by supergravity and superstrings. The inflaton field features a runaway potential with a kinetic pole at the origin that generates the inflationary plateau. After the field rolls over the edge of this plateau, it becomes kinetically dominated, driving a period of kination. The rapid roll of the inflaton is halted, when it crosses an enhanced symmetry point (ESP), where its kinetic density is transferred to the generation of the thermal bath of the hot big bang, through interaction with the Peccei-Quinn (PQ) field. Thereby, the roll of the inflaton is stopped before it travels over superPlanckian distances in field space, which would otherwise undermine the validity of the scalar potential. Trapping the field at the ESP not only reheats the Universe but also ensures that the field becomes heavy and does not give rise to the 5 th force problem, which typically plagues quintessence models. The residual potential density of the field can explain dark energy without resorting to a nonzero value of the cosmological constant. Another aspect of our model which significantly differs from other quintessential inflation models in the literature is that radiation production occurs at reheating and not before, meaning that there is no subdominant thermal bath during kination. ${ }^{8}$

We have studied in detail how the kinetic density of the inflaton is transferred to radiation through the tachyonic and parametric resonant production of PQ particles. Coupling the inflaton to the PQ field is aligned with the economy philosophy of quintessential inflation, in that no arbitrary new field is introduced by hand to interact with the inflaton field responsible for both inflation and dark energy, but the field considered (the PQ field) is already envisaged by beyond the standard model physics to account for the

\footnotetext{
${ }^{8}$ Except due to gravitational particle production $[17,18]$.
}

strong $C P$ problem of $\mathrm{QCD}$ and for the dark matter in the Universe. Moreover, in our model, the interaction between the inflaton and the PQ field ensures that the PQ symmetry is restored during inflation. As a result, the axion field does not exist during inflation, so it does not obtain a superhorizon spectrum of perturbations of its expectation value. This means that there is no issue of axion isocurvature perturbations, which can otherwise be a concern when considering axionic dark matter. ${ }^{9}$

Our model manages to account for observations with natural values of the model parameters. The inflationary observables obtained (see Table I) are in excellent agreement with the Planck satellite findings and are rather robust, in that they do not significantly depend on the location of the ESP down the runaway inflaton direction. This is not surprising, given that the inflationary plateau is generated by the presence of a kinetic pole. For dark energy, the strength of the exponential characterizing the potential slope only implies that the inflaton is suppressed by the grand unified theory (GUT) scale.

All in all, we have presented a new quintessential inflation model which successfully accounts for inflation and dark energy and may well have a basis in fundamental physics.

\section{ACKNOWLEDGMENTS}

K. D. is supported (in part) by the Lancaster-ManchesterSheffield Consortium for Fundamental Physics under the Science and Technology Facilities Council (STFC) Grant No. ST/L000520/1. M. K. is supported by the Communidad de Madrid "Atracción de Talento investigador" Grant No. 2017-T1/TIC-5305 and MINECO (Spain) project FIS2016-78859-P(AEI/FEDER, UE). C. O. is supported by the Faculty of Science and Technology (FST) of Lancaster University.

\footnotetext{
${ }^{9}$ At the breaking of the PQ symmetry the Kibble mechanism may give rise to axionic cosmic strings, which however are harmless since their tension is at the PQ scale $G \mu \sim\left(f / m_{\mathrm{Pl}}\right)^{2} \sim$ $10^{-12}$, so they do not introduce any dangerous signals in the CMB.
}

[1] A. A. Starobinsky, A new type of isotropic cosmological models without singularity, Phys. Lett. 91B, 99 (1980).

[2] K. Sato, Cosmological baryon number domain structure and the first order phase transition of a vacuum, Phys. Lett. 99B, 66 (1981); Adv. Ser. Astrophys. Cosmol. 3, 134 (1987).

[3] D. Kazanas, Dynamics of the universe and spontaneous symmetry breaking, Astrophys. J. 241, L59 (1980).

[4] A. H. Guth, The inflationary universe: A possible solution to the horizon and flatness problems, Phys. Rev. D 23, 347 (1981).
[5] S. Weinberg, The cosmological constant problem, Rev. Mod. Phys. 61, 1 (1989).

[6] L. H. Ford, Cosmological constant damping by unstable scalar fields, Phys. Rev. D 35, 2339 (1987).

[7] C. Wetterich, Cosmology and the fate of dilatation symmetry, Nucl. Phys. B302, 668 (1988).

[8] B. Ratra and P. J. E. Peebles, Cosmological consequences of a rolling homogeneous scalar field, Phys. Rev. D 37, 3406 (1988). 
[9] P. J. E. Peebles and A. Vilenkin, Quintessential inflation, Phys. Rev. D 59, 063505 (1999).

[10] C. Wetterich, Cosmon inflation, Phys. Lett. B 726, 15 (2013).

[11] K. Dimopoulos and C. Owen, Quintessential inflation with $\alpha$-attractors, J. Cosmol. Astropart. Phys. 06 (2017) 027.

[12] K. Dimopoulos, Leonora Donaldson Wood, and Charlotte Owen, Instant preheating in quintessential inflation with $\alpha$-attractors, Phys. Rev. D 97, 063525 (2018).

[13] M. W. Hossain, R. Myrzakulov, M. Sami, and E. N. Saridakis, Unification of inflation and dark energy $\tilde{A}$ la quintessential inflation, Int. J. Mod. Phys. D 24, 1530014 (2015).

[14] C.-Q. Geng, M. W. Hossain, R. Myrzakulov, M. Sami, and E. N. Saridakis, Quintessential inflation with canonical and noncanonical scalar fields and Planck 2015 results, Phys. Rev. D 92, 023522 (2015).

[15] K. Dimopoulos, The curvaton hypothesis and the etaproblem of quintessential inflation, with and without branes, Phys. Rev. D 68, 123506 (2003).

[16] P. A. R. Ade et al., Planck 2015 results. XX. Constraints on inflation, Astron. Astrophys. 594, A20 (2016).

[17] L. H. Ford, Gravitational particle creation and inflation, Phys. Rev. D 35, 2955 (1987).

[18] E. J. Chun, S. Scopel, and I. Zaballa, Gravitational reheating in quintessential inflation, J. Cosmol. Astropart. Phys. 07 (2009) 022.

[19] G. N. Felder, L. Kofman, and A. D. Linde, Instant preheating, Phys. Rev. D 59, 123523 (1999).

[20] A. H. Campos, H. C. Reis, and R. Rosenfeld, Preheating in quintessential inflation, Phys. Lett. B 575, 151 (2003).

[21] B. Feng and M.-z. Li, Curvaton reheating in nonoscillatory inflationary models, Phys. Lett. B 564, 169 (2003).

[22] J. C. B. Sanchez and K. Dimopoulos, Curvaton reheating allows TeV Hubble scale in NO inflation, J. Cosmol. Astropart. Phys. 11 (2007) 007.

[23] K. Dimopoulos and T. Markkanen, Non-minimal gravitational reheating during kination, J. Cosmol. Astropart. Phys. 06 (2018) 021.
[24] T. Opferkuch, P. Schwaller, and B. A. Stefanek, Ricci reheating, J. Cosmol. Astropart. Phys. 07 (2019) 016.

[25] J. C. B. Sanchez and K. Dimopoulos, Trapped quintessential inflation, Phys. Lett. B 642, 294 (2006); 647, 526(E) (2007).

[26] J. C. B. Sanchez and K. Dimopoulos, Trapped quintessential inflation in the context of flux compactifications, J. Cosmol. Astropart. Phys. 10 (2007) 002.

[27] L. Kofman, A. D. Linde, X. Liu, A. Maloney, L. McAllister, and E. Silverstein, Beauty is attractive: Moduli trapping at enhanced symmetry points, J. High Energy Phys. 05 (2004) 030.

[28] R. D. Peccei and H. R. Quinn, Constraints imposed by $C P$ conservation in the presence of instantons, Phys. Rev. D 16, 1791 (1977).

[29] R. D. Peccei and H. R. Quinn, $C P$ Conservation in the Presence of Instantons, Phys. Rev. Lett. 38, 1440 (1977).

[30] S. M. Carroll, Quintessence and the Rest of the World, Phys. Rev. Lett. 81, 3067 (1998).

[31] C. Wetterich, Quintessence: The fifth force, J. Phys. 3N12, 43 (2004).

[32] B. S. Acharya, A. Maharana, and F. Muia, Hidden sectors in string theory: Kinetic mixings, fifth forces and quintessence, J. High Energy Phys. 03 (2019) 048.

[33] H. Baer, K.-Y. Choi, J. E. Kim, and L. Roszkowski, Dark matter production in the early Universe: Beyond the thermal WIMP paradigm, Phys. Rep. 555, 1 (2015).

[34] B. Spokoiny, Deflationary universe scenario, Phys. Lett. B 315, 40 (1993).

[35] M. Joyce and T. Prokopec, Turning around the sphaleron bound: Electroweak baryogenesis in an alternative postinflationary cosmology, Phys. Rev. D 57, 6022 (1998).

[36] J. F. Dufaux, G. N. Felder, L. Kofman, M. Peloso, and D. Podolsky, Preheating with trilinear interactions: Tachyonic resonance, J. Cosmol. Astropart. Phys. 07 (2006) 006.

[37] L. Kofman, A. Linde, and A. A. Starobinsky, Towards the theory of reheating after inflation, Phys. Rev. D 56, 3258 (1997).

[38] Y. Akrami et al., Planck 2018 results. X. Constraints on inflation, arXiv:1807.06211. 